\title{
Entrelacs
}

Cinéma et audiovisuel

14 | 2018

Marchés du film : évolutions, mutations et perspectives

\section{Le Film Bazaar : un marché de projets pour le développement du cinéma indépendant en Inde}

Mélanie Le Forestier

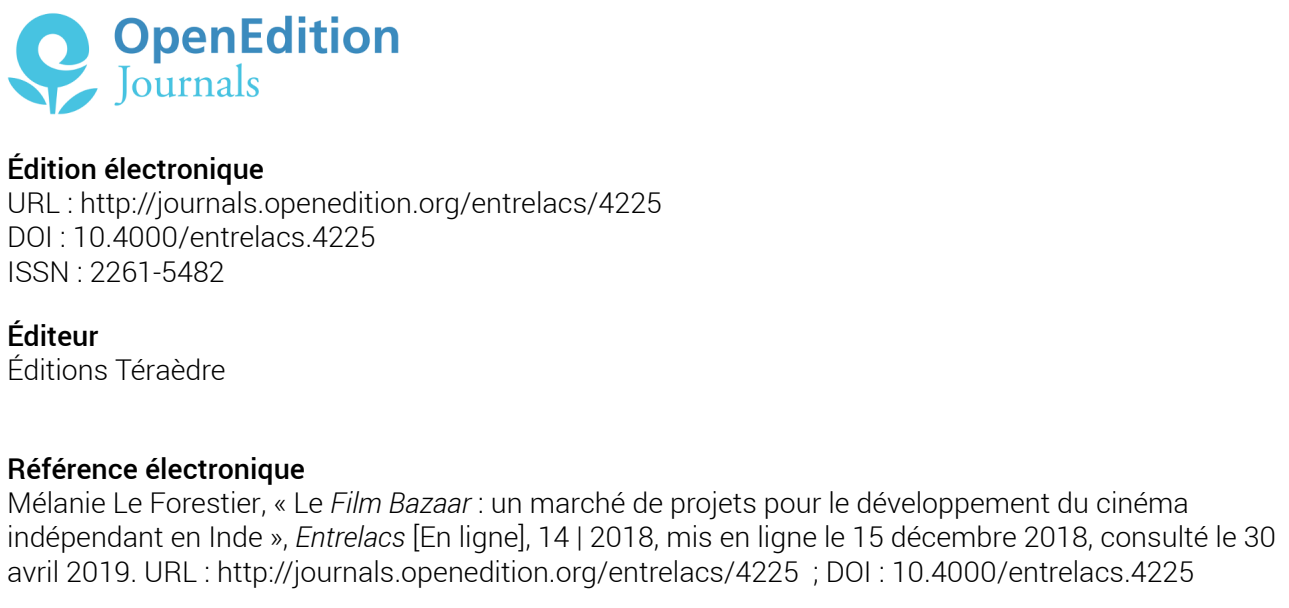

Ce document a été généré automatiquement le 30 avril 2019

Tous droits réservés 


\title{
Le Film Bazaar : un marché de projets pour le développement du cinéma indépendant en Inde
}

\author{
Mélanie Le Forestier
}

1 La production cinématographique indienne est constituée de plusieurs industries régionales indépendantes les unes des autres et ancrées dans des contextes linguistiques et socioculturels différents. L'Inde est aussi la plus grande productrice de films dans le monde, avec presque deux mille long-métrages produits par an selon les dernières études de l'observatoire européen de l'audiovisuel en 2016. Cependant, la place de la production cinématographique indienne "dans le marché cinématographique mondial reste marginale ${ }^{1}$, les exportations ne comptant seulement que pour $7 \%$ de ses recettes totales en $2015^{2}$. Les films indiens, tournés dans neuf langues indiennes principales ${ }^{3}$, dominent quant à eux le marché domestique et se partagent entre $85 \%$ et $90 \%$ des recettes nationales annuelles ${ }^{4}$. Derrière cette forte productivité et diversité linguistique sur le plan quantitatif se cache toutefois une importante inégalité entre les films indiens en termes de financement, production, distribution et médiatisation. Dans les discours académiques et médiatiques, il existe une distinction entre le cinéma hindi, reconnu comme le cinéma dominant en Inde ${ }^{5}$, et les cinémas tournés dans d'autres langues regroupées dans la catégorie " cinémas régionaux ${ }^{6}$ » mais aussi entre films commerciaux et films indépendants. S'il n'existe pas de données quantitatives précises sur le nombre de films "indépendants» produits chaque année, nous avons montré au cours d'une recherche doctorale ${ }^{7}$ que cette pratique artistique alternative a connu un renouveau dans les années 2000 après une première vague dans les années 1960. Dans cet article, nous souhaitons questionner le rôle tenu par le Film Bazaar (FB), unique marché du film en Inde dans le développement du cinéma indépendant contemporain (des années 2000 à nos jours). Phénomène encore peu étudié dans le champ des études cinématographiques, puisqu'à notre connaissance il n'existe à ce jour aucune analyse fine du FB, notre objectif est de comprendre quelle place il occupe dans le paysage cinématographique indien. 


\section{Une approche multidimensionnelle du marché du film}

2 Dans le champ des études cinématographiques, le marché du film a souvent été étudié depuis l'approche économique et marketing du cinéma. Dans ce cadre, il se définit comme un « lieu de rencontre entre l'offre et la demande ${ }^{8}$ ", une manifestation concrète d'un «mécanisme abstrait ${ }^{9}$ » et complexe d'échanges nationaux et internationaux. Pour Jérôme Paillard, directeur délégué du Marché du Film du Festival de Cannes, le marché est traditionnellement un «salon professionnel $1^{10}$ ", « où se rencontrent des vendeurs qui ne sont pas les producteurs, mais des sociétés spécialisées qui agrègent des films de plusieurs producteurs (entre 15 et 40 ) et qui ont des mandats pour les vendre - et des acheteurs. ${ }^{11}$ » De manière générale, un marché du film peut être associé à un festival de cinéma (le Marché du Film du Festival de Cannes, l'European Film Market de La Berlinale), ou être un événement à part entière (l'American Film Market à Los Angeles). Le FB correspond quant à lui à un "marché de projets de films" ou "pré-marché ${ }^{12}$. Il se concentre donc plus sur le développement et la production que sur l'achat-vente de films.

Dans cette perspective, notre objectif est de dépasser une approche centrée sur la dimension commerciale pour examiner le $\mathrm{FB}$ comme un phénomène multidimensionnel. Notre cadre méthodologique s'appuie sur-le-champ des recherches sur les festivals de cinéma ${ }^{13}$ qui intègre les travaux sur les marchés du film et les fonds de financement. À l'instar des festivals de cinéma, les marchés s'inscrivent plus largement dans « un circuit de marchés audiovisuels internationaux ${ }^{14} »$. Nous retiendrons ici deux principaux thèmes développés dans ces recherches. Le premier thème est celui de l'évolution du rôle joué par ces festivals. Alors qu'ils étaient principalement considérés comme des plateformes

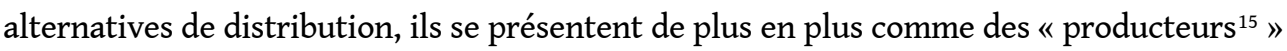
des cinémas du monde en facilitant le financement, la production, la programmation et distribution des films sélectionnés. De ce fait, les festivals de cinéma, mais aussi les marchés du film, agissent comme des "intermédiaires culturels ${ }^{16}$ » et développent une « logique commerciale et [une] logique de reconnaissance symbolique, en s'appuyant à la fois sur le film comme produit culturel et sur le film comme produit économique et médiatique ${ }^{17}$ ». Le FB se définit donc comme un "espace mixte ${ }^{18}$ », concept qu'emploie Janet Harbord pour définir un festival de cinéma comme étant à la fois « un marché et un forum d'évaluation esthétique ${ }^{19}$ ». Le second thème est celui de l'articulation entre des dynamiques régionales, nationales et transnationales. Comme le rappelle Marijke De Valck, les festivals se construisent comme des espaces comportant «à la fois des composants locaux et internationaux ${ }^{20} »$. Il en va de même pour les marchés du film qui se caractérisent comme des «espaces de flux ${ }^{21}$ » créant des réseaux d'interrelations et interconnexions entre professionnels du cinéma. Si Romain Lecler met en évidence que ce circuit est «moins géographique que chronologique ${ }^{22}$ » et cyclique, nous focaliserons malgré tout notre attention sur la situation géographique du FB puisque notre analyse montre que ce marché du film s'avère décentré, autant dans le paysage cinématographique indien que dans l'économie du cinéma mondial. Le FB s'inscrit dans "un système international polycentrique et hiérarchisée ${ }^{23}$ ", mais contrairement à d'autres festivals en Asie (comme le Pusan International Film Festival, Tokyo International Film Festival ou Hong Kong International Film Festival), son objectif n'est pas d'être une plateforme centrale au sein du marché mondial, mais plutôt d'être un « lieu noda ${ }^{24}$ » pour le cinéma indépendant indien. Ces différents événements du côté de 
l'Asie ont néanmoins en commun d'avoir réussi à devenir «des lieux majeurs de la circulation marchande des biens symboliques ${ }^{25}$ » en Asie et dans le monde.

Nous souhaitons donc dans cet article étudier le FB comme une instance où se côtoient et se négocient « un espace marchand et un espace symbolique ${ }^{26}$ ", non seulement traversé par une «double visée, culturelle et commerciale ${ }^{27}$ », mais aussi par des dynamiques locales/globales. Notre démarche méthodologique implique un rapprochement entre Cultural Studies et économie politique critique de la communication ${ }^{28}$ qui permet «l'articulation entre culture et économie, entre analyse des discours et celle des industries, dispositifs et équipements culturels ${ }^{29} »$. Nous avons ici privilégié une approche discursive avec un corpus constitué de 659 documents comprenant d'un côté les archives des documents publiés par le FB et la National Film Development Corporation (agence en charge de l'organisation du marché), et d'un autre côté les discours médiatisés des acteurs de ce marché (organisateurs, producteurs, distributeurs, réalisateurs) dans la presse quotidienne indienne et sur sept sites Internet indiens consacrés au cinéma indépendant ${ }^{30}$. Le choix d'analyser les discours médiatisés s'explique par le fait que la presse reste la principale forme médiatique qui représente et rend compte du marché comme une "expérience suivie en direct [...], instillant une forme d'authenticité à l'événement ${ }^{31} »$. Les sites Internet et blogs consacrés au cinéma indépendant indien inclus dans le corpus constituent quant à eux un espace d'expression privilégié pour les cinéastes indépendants indiens ${ }^{32}$.

\section{Marché du film et cinéma indépendant indien}

Dans le cadre de notre travail doctoral, nous avons mis en évidence la situation marginale du cinéma indépendant au sein du paysage cinématographique indien. L'expression " cinéma indépendant » renvoie tout autant à une pratique cinématographique en marge des logiques industrielles dominantes qu'à des films qui s'éloignent des standards narratifs et stylistiques des films produits par Bollywood. L'économie du cinéma indépendant indien est cependant fragile, car il n'existe pas d' " espace alternatif ${ }^{33}$ ", selon le cinéaste Amartya Bhattacharyya (Capital I, 2014), qui soit consacré au développement et au soutien d'une production indépendante. Le cinéma n'a jamais été la priorité du gouvernement indien ${ }^{34}$ et il n'y a pas de système institutionnel de subventions. L'industrie du cinéma est l'un des secteurs les plus taxés en Inde avec l'industrie du tabac, mais comme le précise Kiran Rao (réalisatrice de Dhobi Ghat en 2010), ces revenus ne sont pas réinvestis dans le fonctionnement de l'industrie cinématographique ${ }^{35}$. À l'instar du réalisateur Ritesh Batra (The Lunchbox 2010), les cinéastes indépendants insistent sur l'idée du développement nécessaire d'un " écosystème » qui permettrait de soutenir et pérenniser le cinéma indépendant en Inde. Celui-ci serait constitué «de studios/ financeurs, de maisons de production, de cinéastes avec une voix réellement indépendante, de programmes de développement de talents, de festivals avec un vrai pouvoir de programmation, de lieux dédiés aux films indépendants, une presse écrite pour le cinéma indépendant [et] des organismes dédiés au développement d'un public. ${ }^{36}$ »

Le FB constitue l'une des rares propositions du gouvernement indien pour soutenir le développement et la production de films indépendants. C'est un marché du film créé en 2007 à l'initiative de Nina Lath Gupta, alors directrice de la National Film Development Corporation of India (NFDC). Sa particularité est d'être un événement culturel institutionnel puisque la NFDC est une agence fondée en $1960^{37}$ par le Ministère de l'Information et de 
l'Audiovisuel (Ministry of Information and Broadcasting). Son « objectif principal [était] la promotion du Mouvement du Bon Cinema ${ }^{38} »-$ expression utilisée pour désigner le cinéma parallèle, ou le nouveau cinéma indien apparu dans les années 1950. La création du FB correspond à une période de renouvellement de la NFDC dans les années 2000, à une époque où l'agence fut menacée d'être fermée en raison de difficultés financières. Le marché répond donc aux missions de la NFDC qui sont d'œuvrer à «la promotion des films, la distribution, la formation, la vente, la restauration et le développement ${ }^{39}$ ». Il s'agit également de contribuer au développement d'« un cinéma d'auteurs fidèles à des critères de qualité esthétique et de pertinence sociale, de maintenir une certaine pluralité du cinéma indien en finançant des projets provenant des diverses régions du pays ${ }^{40}$ ». Il a aussi pour vocation de développer "des collaborations entre l'Inde et l'industrie cinématographique mondiale dans le domaine de la production et de la distribution ${ }^{41} »$.

7 Le marché se présente comme un "forum de coproduction ${ }^{42}$ ", car ses activités s'étendent, dès la seconde édition, au développement de scénarios ainsi qu'à des ateliers d'aide à la production. Dans son format actuel, le FB compte une dizaine d'activités dont les principales sont la présentation de projets en recherche de co-production (Coproduction Market), l'aide au développement de scénario (Screenwriters' Lab), la présentation de films en cours de montage (Work-in-Progress Lab), la projection de films avec les Industry Screenings et la Viewing Room (bibliothèque numérique de films en recherche de distributeur), des ateliers de formation pour les producteurs (Producers' Lab) ainsi que des tables rondes et conférences sur divers aspects du secteur (Knowledge Series). Cette diversité du programme participe à la « matérialité hybride ${ }^{43}$ » du marché. Son rôle d'intermédiaire culturel est aussi renforcé par sa proximité avec l'International Film Festival of India (IFFI). Ses deux « événements culturels ${ }^{44}$ » sont organisés en même temps (en novembre) et dans la même ville (Panaji). Si ces deux manifestations furent longtemps indépendantes l'une de l'autre, autant dans leur organisation que dans leur financement, leur rapprochement s'est intensifié en 2017 quand le Ministère de l'Information et de l'Audiovisuel a décidé que la NFDC serait désormais aussi responsable de l'organisation du Festival au lieu du Directorate of Film Festival (autre service du Ministère).

\section{Un marché de projets doublement décentré}

Le caractère institutionnel de la NFDC et du FB ne cache pas leur situation marginale au sein du marché cinématographique national. Le marché du film a lieu à Panaji, capitale de l'État de Goa. Le choix du lieu d'organisation est avant tout pragmatique et s'explique par la présence de l'IFFI dans la même ville depuis 2004. Le FB se retrouve donc fortement décentré par rapport aux capitales cinématographiques indiennes comme Mumbai ou Hyderabad. Ce décentrement par rapport aux centres de production cinématographique indienne est une façon de faire valoir la diversité cinématographique en Inde sans privilégier une des capitales culturelles et industries régionales indiennes. La situation du FB se distingue aussi de " la relation importante entre les villes et le circuit festivalier ${ }^{45}$ » qui est le plus souvent privilégiée. Julian Stringer a en effet démontré que ce sont les villes qui agissent comme des " points nodaux dans ce [circuit festivalier international], et non les industries du cinéma ${ }^{46} »$. Pourtant, Panaji est pratiquement absente des discours et documents officiels du FB et des articles de presse.

Le FB se trouve également décentré par rapport aux marchés cinématographiques internationaux. Un des objectifs du marché est d'« encourager des collaborations entre 
l'industrie internationale du cinéma et celle de l'Asie du Sud ${ }^{47}$ » dès les stades initiaux des projets et non pas uniquement au moment de leurs distributions ${ }^{48}$. Pour la productrice Guneet Monga (Sikhya Entertainment), il s'agit de faire venir les acteurs du marché mondial « intéressés par les films indiens ${ }^{49}$ » directement en Inde. Cette stratégie facilite selon elle le développement de projets indiens alors qu'il serait plus difficile «d'attirer l'attention [...] sur le cinéma indien quand vous êtes à Berlin, Toronto ou Cannes ${ }^{50}$ ». Le nombre de participants (porteurs de projets, mentors, producteurs et distributeurs indiens et internationaux) n'a cessé d'augmenter chaque année, passant de 170 participants de 11 pays différents en $2007^{51}$ à 1206 participants de 38 pays différents en 2016. Cette évolution montre que le $\mathrm{FB}$ a réussi à attirer les producteurs et distributeurs du monde entier, même si ces résultats sont modestes comparés à ceux du Marché du film du Festival de Cannes par exemple, où plus de 12000 professionnels s'y rendent en moyenne chaque année ${ }^{52}$. Cela témoigne néanmoins du dynamisme du FB et de sa capacité à s'implanter dans un système transnational en seulement dix ans.

\section{Un espace de résistance et de négociation}

10 L'évolution et le dynamisme du FB participent à en faire un "lieu de pouvoir ${ }^{53}$ " alternatif. Il permet aux cinéastes indépendants de trouver un espace d'échanges et de production où il leur est possible de résister au "pouvoir homogénéisant ${ }^{54}$ » des industries cinématographiques indiennes. De fait, le FB ne peut pas être considéré comme un espace « neutre ${ }^{55}$ ». Il se positionne dans le paysage cinématographique indien comme un défenseur d'une diversité cinématographique, autant d'un point de vue culturel et linguistique qu'économique et stylistique. Cependant, il se constitue aussi comme un espace de négociation. D'un point de vue économique, plusieurs studios de production bollywoodiens ont commencé à coproduire ou distribuer des films présentés au FB dans les années 2010. C'est le cas par exemple de la société Yash Raj Films, une des principales productrices des succès commerciaux des années 1990, qui fut coproductrice du film Titli réalisé par Kanu Behl (2013) et de Dum Laga Ke Haisha de Sharat Katariya (2015).

11 Sur le plan stylistique, plusieurs cinéastes comme Bikas Mishra (Chauranga 2010) ou Onir ( I Am 2010) avaient au départ des doutes quant à la capacité de mentors étrangers (des producteurs et distributeurs indiens ou internationaux expérimentés qui aident au développement des films) de "réellement comprendre le contenu et le contexte du cinéma $^{56}$ " indépendant indien. Leurs mentors les aidèrent cependant à retravailler et affiner les aspects techniques et le contenu de leur scénario tout en respectant la liberté artistique des cinéastes. La productivité et la richesse de ces rencontres est une des principales thématiques qui ressort des discours des cinéastes. Pour Bikas Mishra par exemple, sa collaboration avec les autres participants sélectionnés et son mentor Marten Rabarts, alors directeur de Binger Filmlab ${ }^{57}$, fut déterminante dans la réussite de son premier long-métrage Chauranga : "Il y avait une discussion intelligente et très saine entre scénaristes, et cela nous valorisait en créant un environnement où les scénaristes pouvaient réellement contribuer de manière constructive aux écrits de chacun ${ }^{58}$ ».

D'autres cinéastes pointent cependant comme un des risques possibles de ces collaborations internationales le fait de voir des films sélectionnés uniquement parce qu'ils répondraient « aux traditions réalistes européennes ${ }^{59}$ » ou d'être ensuite labellisés «films de festival»- compris dans ce cadre non pas comme des « films présentés dans des festivals », mais comme des films «supposément produits pour les festivals ${ }^{60}$ ». On 
retrouve cette idée d'influence sur les choix artistiques des cinéastes dans les propos de Nina Lath Gupta concernant deux principaux critères de sélection des projets: des contenus de «bonne qualité [et] la capacité à bien raconter une histoire ${ }^{61}$ » ainsi qu'une "viabilité internationale ${ }^{62}$ ». Ce dernier point est particulièrement important, car les films indépendants n'ont que très peu de chance d'être distribués dans les salles de cinéma indienne ${ }^{63}$. Par conséquent, les festivals de cinéma internationaux constituent une plateforme de diffusion essentielle. En retour, la sélection dans des festivals de renommée internationale contribue au rayonnement des films au sein du marché national indien. Cette dimension est valorisée dans chaque catalogue annuel du FB où une à deux pages sont consacrées aux films qui ont été sélectionnés dans des festivals internationaux majeurs comme le Festival de Cannes (Miss Lovely en 2012, The Lunchbox en 2013, Titli en 2014, Chauthi Koot et Masaan en 2015), la Mostra de Venise (Court en 2014), la Berlinale ( Newton en 2017), le Festival International du film de Toronto (Ship of Theseus en 2012, Margarita with a Straw en 2014) ou encore le Festival du film de Sundance (Liar's Dice en 2014). En tout, vingt-huit films indiens ont rencontré un succès national et international ${ }^{64}$ . Le FB joue donc un rôle actif dans le renouvellement et la diversité de la production cinématographique indienne. Plus d'une centaine de films est présentée chaque année dans les différents programmes du FB, même si moins de dix films sont choisis pour les ateliers d'aides au développement et moins d'une trentaine pour la coproduction. C'est peu par rapport à la production annuelle de films sur l'ensemble du territoire national. Pour autant, le principal objectif du marché n'est pas tant quantitatif que qualitatif. Il s'agit plutôt de se focaliser sur l'accompagnement d'un nombre restreint de projets afin qu'ils aboutissent et puissent « percer sur le marché international et le circuit festivalier ${ }^{65}$ ».

\section{Conclusion}

13 En focalisant notre attention sur la situation du FB au sein des marchés cinématographiques indiens et internationaux, nous avons cherché à comprendre quel rôle joue ce marché du film dans le développement d'un cinéma indépendant contemporain en Inde. De par son ancrage dans un circuit d'échanges internationaux, il participe à « une arène culturelle unique qui agit comme une zone de contact pour faire face aux relations de pouvoir inégales ${ }^{66} \%$. Dans ce cadre, nous pouvons remarquer que les stratégies de résistance et de négociation des cinéastes indépendants par rapport aux industries cinématographiques indiennes prennent une dimension inévitablement transnationale. Un processus de déterritorialisation de la production des films, suivi d'une première diffusion à l'international, est nécessaire pour avoir une chance d'être distribué sur le territoire national. S'ils sont peu nombreux, la présence de ces films dans des lieux majeurs de la circulation internationale des biens symboliques contribue indirectement à faire de ce cinéma indépendant un « ambassadeur culturel ${ }^{67} »$ de l'Inde. Il reste que la question de la distribution des films indépendants dans les salles de cinéma indiennes continue d'être un problème majeur pour les cinéastes indépendants qui souhaitent avant tout montrer leurs films aux spectateurs indiens ${ }^{68}$.

14 De même, les actions du FB ne peuvent empêcher la récupération du label «cinéma indépendant » par les studios bollywoodiens qui l'utilisent désormais pour désigner des films qu'ils produisent à plus petit budget. L'un des enjeux majeurs actuels pour les cinéastes indépendants est de réussir à négocier l'autonomie et indépendance de leur 
pratique artistique face à une industrie tentaculaire et insatiable. Le FB n'est quant à lui pas exempt de tout risque, car la NFDC, en tant qu'organisme institutionnel, est soumise aux aléas de la scène politique nationale. Après avoir dirigé la NFDC depuis 2006, Nina Lath Gupta a par exemple été renvoyée par la Ministre de l'Information et de l'Audiovisuel en mars 2018. Avec ce départ précipité, une page importante se tourne pour la NFDC, mais aussi pour le FB dont Nina Lath Gupta avait été l'instigatrice. Le rôle de l'agence dans la production de films indépendants demeure d'autant plus incertain que la NFDC n'est plus dans la capacité depuis quelques années de produire des films par manque de capitaux ${ }^{69}$.

\section{Liste des abréviations}

AFM : Asian Film Market

BIFF/PIFF : Busan International Film Festival/Pusan International Film Festival

IFFI : International Film Festival of India

NFDC : National Film Development Corporation

\section{Corpus}

Ritesh Batra, « The Indian Independent Film Industry: Where Do We Go Now? », Truly Free Film, 2012.

Amartya Bhattacharyya, «Independent Cinema Is The Only Way », India Independent Films, 2017.

Onir Dhar, «Petition: Save Indie Cinema »: https://www.change.org/p/save-indiecinema

Aditi Dharmadhikari, « 10 Years/10 Films: The Best of Film Bazaar's Screenwriters' Lab », Pandolin, 22 février 2016.

Film Bazaar, Film Bazaar Project 2015.

Film Bazaar, Co-Production Market Report 2016.

Charmy Harikrishnan, «NFDC's FB becomes indie cinema's go-to place for funds », The Economic Times, 27 novembre 2016.

Vanita Kohli-Khandekar, « NFDC writes a new script for Indian cinema », Business Standard , 2014.

Udita Jhunjhunwala, « Nina Lath Gupta: The 10-year-old journey of FB », Livemint, 2016.

NFDC, Annual Report 2008-2009.

Nandini Ramnath, "'The films we now make get funding in the market' ", Livemint, 19 novembre 2012.

Liz Shackleton, « India's international horizons », Screen Daily, 19 novembre 2012

Suhani Singh, « Truly independent cinema is all about invention, innovation and artistic inquiry », India Today, 2013.

Shweta Wadhwa, «Film Bazaar Has Changed The Pattern of Cinema Being Produced in the Country », Pandolin, 2015. 


\section{BIBLIOGRAPHIE}

Soojeong Ahn, The Pusan International Film Festival, South Korean Cinema and Globalization, Hong Kong University Press, 2012.

Pierre-Jean Benghozi, Claire Nénert, « Création de valeur artistique ou économique : Festival International du film de Cannes au marché du film », Recherche et Applications en Marketing, Vol. 10, No. 4, pp. 65-76, 1995.

Laurent Creton, L'économie du cinéma en 50 fiches, Armand Colin, 2012.

Bernard Darras, «"Les études culturelles sont-elles solubles dans les Cultural Studies ?” par Marie-Hélène Bourcier, François Cusset et Armand Mattelart », MEI, n²4-25, 2006.

François Da Silva, « Acheter et coproduire avec l'Amérique du sud et l'Asie », in Claude Forest (dir.), L'internationalisation des productions cinématographiques audiovisuelles, Paris, Presses Universitaires du Septentrion, 2017.

Camille Deprez, Bollywood : cinéma et mondialisation, Presses Universitaires du Septentrion, 2010.

Marijke De Valck, Films Festivals. From European Geopolitics to Global Cinephilia, Amsterdam University Press, 2007.

Marijke De Valck, Brendan Kredell, Skadi Loist (Éd.), Film Festivals: History, Theory, Method, Practice, Routledge, 2016.

Tamara L. Falicov, « "The Festival Film”: Film Festivals as Cultural Intermediaries », in Marijke De Valck, Brendan Kredell, Skadi Loist (Éd.), Film Festivals: History, Theory, Method, Practice, Routledge, 2016, pp. 209-229.

Marie-France García-Parpet, Romain Lecler, Gustavo Sorá, « Foires, salons et marchés internationaux. Circulation des biens symboliques et mondialisation des places marchandes » in Johanna Siméant (Éd.), Guide de l'enquête globale en sciences sociales, CNRS Éditions, 2015, pp. 95-113.

K. Moti Gokulsing, Wimal Dissayanake (dir.), Routledge Handbook of Indian Cinemas, New York, Routledge, 2013.

Janet Harbord, Film Cultures, Londres, Thousand Oaks et New Delhi, Sage Publications, 2002.

Mélanie Le Forestier, Imaginaires nationaux et dynamiques transnationales : étude du cinéma hatke en Inde, Thèse de doctorat soutenue à l'Université de Toulouse 2, 2016.

Skadi Loist, " The film festival circuit: Networks, hierarchies, and circulation », in Marijke De Valck, Brendan Kredell, Skadi Loist (Éd.), Film Festivals: History, Theory, Method, Practice, Londres, New York, Routledge, 2016, pp. 49-63.

Éric Maigret, Franck Rebillard (dir.), «Cultural Studies et économie politique de la communication », Réseaux, vol. 4, n 192, 2015.

Abé Markus Nornes, Cinema Babel. Translating Global Cinema, University of Minnesota Press, 2007 . 
Jérôme Paillard, « Le Marché du Film du festival de Cannes, événement majeur de l'industrie cinématographique », Géo-économie, vol. 58, no. 3, 2011, p. 77-87.

Amanda Rueda, «Festival de cinéma : Médiations et construction de Territoires imaginaires », Culture \& Musées, $\mathrm{n}^{\circ}$ 14, 2009, pp. 149-171.

Daniel Steinhart, « Fostering International Cinema: The Rotterdam Film Festival, CineMart, and Hubert Bals Fund », Mediascape, $n^{\circ}$ 2, 2006.

Julian Stringer, « Global Cities and the International Film Festival Economy », in Shiel M., Fitzmaurice T. (Éd.), Cinema and the City: Film and Urban Societies in a Global Context, Blackwell, 2001, pp. 134-144.

Daya Thussu, Communicating India's Soft Power: Buddha to Bollywood, New York, Palgrave Macmillan, 2013.

Antonio Vassilis, « Les puissances émergentes dans la bataille mondiale de l'attraction : Bollywood, vecteur du soft power de l'Inde? », Revue Interventions économiques, n 55, 2016.

Cindy Hing-Yuk Wong, Film Festivals. Culture, People and Power on the Global Screen, Rutgers University Press, 2011.

\section{NOTES}

1. Antonios Vassilis, "Les puissances émergentes dans la bataille mondiale de l'attraction : Bollywood, vecteur du soft power de l'Inde? ", Revue Interventions économiques, n55, 2016, p. 6.

2. Observatoire européen de l'audiovisuel, FOCUS, Tendances du marché mondial du film, Strasbourg, OEA, 2015.

3. Selon les dernières données du Censor Board of Film Certification.

4. OEA, op.cit.

5. L'industrie du cinéma hindi est surnommée Bollywood depuis les années 1990.

6. K. Moti Gokulsing, Wimal Dissayanake (dir.), Routledge Handbook of Indian Cinemas, New York, Routledge, 2013.

7. Mélanie Le Forestier, Imaginaires nationaux et dynamiques transnationales: étude du cinéma hatke en Inde, Thèse de doctorat soutenue à l'Université de Toulouse 2 Jean Jaurès, 2016.

8. Laurent Creton, L'économie du cinéma en 50 fiches, Paris, Armand Colin, 2012, p. 13.

9. Marie-France García-Parpet, Romain Lecler, Gustavo Sorá, « Foires, salons et marchés internationaux. Circulation des biens symboliques et mondialisation des places marchandes » in Johanna Siméant (Éd.), Guide de l'enquête globale en sciences sociales, Paris, CNRS Éditions, 2015, p. 95.

10. Jérôme Paillard, «Le Marché du Film du festival de Cannes, événement majeur de l'industrie cinématographique », Géo-économie, vol. 58, no. 3, 2011, p. 77.

11. Ibid.

12. Soojeong Ahn, The Pusan International Film Festival, South Korean Cinema and Globalization, Hong Kong University Press, 2012.

13. Voir la bibliographie régulièrement mise à jour du Film Festival Research Network.

14. Romain Lecler, "La montée des marchés à Cannes. Le circuit des échanges audiovisuels internationaux, sa chronologie et ses trajets professionnels ", Réseaux vol.6, n² 200, 2016, p. 228.

15. Daniel Steinhart, «Fostering International Cinema: The Rotterdam Film Festival, CineMart, and Hubert Bals Fund », Mediascape, ${ }^{\circ} 2,2006$, p. 1.

16. Tamara Falicov, “"The Festival Film”: Film Festivals as Cultural Intermediaries », in Marijke De Valck, Brendan Kredell et Skadi Loist (op.cit.), p. 212. 
17. Pierre-Jean Benghozi, Claire Nénert, «Création de valeur artistique ou économique : Festival International du film de Cannes au marché du film », Recherche et Applications en Marketing, Vol. 10, No. 4, 1995, p. 65.

18. Janet Harbord, Film Cultures, Londres, Thousand Oaks et New Delhi, Sage Publications, 2002, p. 60.

19. Ibid., p. 70.

20. Marijke De Valck, Films Festivals. From European Geopolitics to Global Cinephilia, Amsterdam, Amsterdam University Press, 2007, p.135.

21. Harbord, op.cit, p. 59.

22. Ibid., p. 229.

23. Marie-France García-Parpet, Romain Lecler, Gustavo Sorá, op.cit., p. 112.

24. Cindy Hing-Yuk Wong, Film Festivals. Culture, People and Power on the Global Screen, Rutgers University Press, 2011, p. 1.

25. Marie-France García-Parpet, Romain Lecler, Gustavo Sorá, op.cit., p. 112.

26. Amanda Rueda, «Festival de cinéma : Médiations et construction de Territoires imaginaires ", Culture \& Musées, n¹4, 2009, p. 158.

27. Pierre-Jean Benghozi, Claire Nénert, op.cit., p. 65.

28. Éric Maigret, Franck Rebillard (Éd.), «Cultural Studies et économie politique de la communication ", Réseaux, vol. 4, n¹92, 2015.

29. Armand Mattelart, Bernard Darras, "Les études culturelles sont-elles solubles dans les Cultural Studies? par Marie-Hélène Bourcier, François Cusset et Armand Mattelart. Entretiens réalisés par Bernard Darras ", MEI, n²4-25, 2006, p. 19.

30. Il s'agit des sites Passion for Cinema, Dear Cinema, Pandolin, Indian Independent Films, Jamuura, Long Live Cinema et Film Companion.

31. Janet Harbord, op.cit, p. 68.

32. Mélanie Le Forestier, op.cit.

33. Amartya Bhattacharyya, "Independent Cinema Is The Only Way », India Independent Films, 2017.

34. Le cinéma ne fut officiellement reconnu comme industrie par le gouvernement indien qu'en 1998.

35. Suhani Singh, «Truly independent cinema is all about invention, innovation and artistic inquiry », India Today, 2013.

36. Ritesh Batra, «The Indian Independent Film Industry: Where Do We Go Now? », Truly Free Film, 2012.

37. Cette agence, au départ intitulée Film Finance Corporation, fut renommée National Film Development Corporation of India en 1975.

38. NFDC, «FB : About » : https://filmbazaarindia.com/about/.

39. Vanita Kohli-Khandekar, " NFDC writes a new script for Indian cinema », Business Standard, 2014.

40. Camille Deprez, Bollywood : cinéma et mondialisation, Paris, Presses Universitaires du Septentrion, 2010, p. 38.

41. NFDC, Annual Report 2008-2009.

42. François Da Silva, « Acheter et coproduire avec l'Amérique du sud et l'Asie », in Claude Forest (dir.), L'internationalisation des productions cinématographiques audiovisuelles, Paris, Presses Universitaires du Septentrion, 2017, p. 191.

43. Janet Harbord, op.cit, p. 60.

44. Cindy Hing-Yuk Wong, op.cit, p.160.

45. Soojeong Ahn, op.cit, p. 17. 
46. Julian Stringer, « Global Cities and the International Film Festival Economy », in Mark Shiel, Tony Fitzmaurice (Éd.), Cinema and the City: Film and Urban Societies in a Global Context, Malden, Blackwell, 2001, p.138.

47. Film Bazaar, Film Bazaar Project 2015.

48. Nandini Ramnath, "The films we now make get funding in the market ", Livemint, 19 novembre 2012.

49. Liz Shackleton, « India's international horizons », Screen Daily, 19 novembre 2012.

50. Ibid.

51. Selon les rapports annuels du FB.

52. Selon le site officiel du Marché du Film.

53. Abé Mark Nornes, Cinema Babel. Translating Global Cinema, Minneapolis, University of Minnesota Press, 2007, p. 65.

54. Tamara Falicov, op.cit, p.216.

55. Soojeong Ahn, op.cit, p.66.

56. Aditi Dharmadhikari, op.cit.

57. Centre de développement de longs-métrages et de documentaires hollandais soutenant le développement de projets venant du monde entier.

58. Aditi Dharmadhikari, «10 Years/10 Films: The Best of Film Bazaar's Screenwriters' Lab», Pandolin, 22 février 2016.

59. Charmy Harikrishnan, "NFDC's Film Bazaar becomes indie cinema's go-to place for funds ", The Economic Times, 27 novembre 2016.

60. Julian Stringer, cite par Tamara Falicov, op.cit, p.216.

61. Shweta Wadhwa, «Film Bazaar Has Changed The Pattern of Cinema Being Produced in the Country », Pandolin, 2015.

62. Liz Shackleton, op. cit.

63. Mélanie Le Forestier, op.cit.

64. Film Bazaar, Co-Production Market Report 2016.

65. Udita Jhunjhunwala, « Nina Lath Gupta: The 10-year-old journey of FB », Livemint, 2016.

66. Julian Stringer, op.cit, p. 138.

67. Daya Kishan Thussu, Communicating India's Soft Power: Buddha to Bollywood, New York, Palgrave Macmillan, 2013, p.151.

68. Onir Dhar, «Petition : Save Indie Cinema », change.org, octobre 2012 .

69. Udita Jhunjhunwala, op.cit.

\section{RÉSUMÉS}

Dans cet article, nous souhaitons questionner le rôle tenu par le Film Bazaar (FB) dans le développement du cinéma indépendant contemporain en Inde (des années 2000 à nos jours). Présenté comme le "plus grand marché du film en Asie du Sud», il se caractérise comme un espace de médiation institutionnel réunissant des professionnels du cinéma venus du monde entier. L'analyse discursive des documents publiés par le Film Bazaar et du discours des organisateurs et des professionnels du cinéma participant au marché montre qu'à l'instar des festivals de cinéma, le FB se caractérise comme un "marché de projets " traversé par des logiques marchandes et symboliques, mais aussi par des dynamiques locales, nationales et 
internationales. Il se présente comme un «espace mixte » où les cinéastes indépendants de résister et négocier avec les industries cinématographiques indiennes et internationales.

In this paper, we would like to examine the role of the Film Bazaar (FB) in the development of a contemporary independent cinema in India (from the 2000s). As "the largest South Asian film market", Film Bazaar is an institutional space of mediation gathering film professionals from all over the world. The discursive analysis is focusing on the programs and documents published by the market as well as the mediatized discourse of the participants. Following the example of film festivals, it shows that this film market is a "pre-market" or a "project market" crossed by commercial and symbolic logics as well as local, national and international flows. It can be seen as a "mixed space" where independent filmmakers can resist and negotiate with national and international film industries.

\section{AUTEUR}

\section{MÉLANIE LE FORESTIER}

Titulaire d'un doctorat en sciences de l'information et de la communication obtenu en 2016 à l'Université Toulouse 2 - Jean Jaurès. Sa thèse, Imaginaires nationaux et dynamiques transnationales : étude du cinéma hatke en Inde, portait sur l'analyse de la construction contrehégémonique du cinéma indépendant contemporain en Inde. Membre du LERASS (Laboratoire d'Études et de Recherches Appliquées en Sciences Sociales), elle est actuellement post-doctorante «IDEFI DEFI Diversités » au Centre Gaston Berger de l'Institut National des Sciences Appliquées de Toulouse où elle effectue une recherche sur les enjeux de l'interculturalité dans le champ des professionnels des ingénieurs 